\title{
Barriers and facilitators to implementing a mobile intervention with task shifting in the treatment of depressive symptoms in people with diabetes and / or hypertension in Brazil: A study protocol using the CFIR.
}

Hellen Carolina Martins Castro ( $\nabla$ hellenmartins@usp.br)

Universidade de São Paulo https://orcid.org/0000-0001-7865-0941

Marcia Thereza Couto

University of Sao Paulo: Universidade de Sao Paulo

Kate Daley

University of Sao Paulo: Universidade de Sao Paulo

Thais Izabel Ugeda Rocha

University of Sao Paulo: Universidade de Sao Paulo

Suzana Crismanis de Almeida Lopes Aschar

University of Sao Paulo: Universidade de Sao Paulo

Daniela Vera Cruz dos Santos

University of Sao Paulo: Universidade de Sao Paulo

Heloisa Garcia Claro

Universidade Estadual de Campinas - Campus Cidade Universitaria Zeferino Vaz: Universidade Estadual de Campinas

Ricardo Araya

University of Sao Paulo: Universidade de Sao Paulo

Paulo Rossi Menezes

University of Sao Paulo: Universidade de Sao Paulo

Study protocol

Keywords: Mental Health, Depression, Chronic diseases, Mobile Intervention, e-health

Posted Date: October 9th, 2020

DOI: https://doi.org/10.21203/rs.3.rs-88770/v1 
License: (c) (i) This work is licensed under a Creative Commons Attribution 4.0 International License. Read Full License 
Barriers and facilitators to implementing a mobile intervention with task shifting in the treatment of depressive symptoms in people with diabetes and / or hypertension in Brazil: A study protocol using the CFIR.

Authors:

Castro, Hellen Carolina Martins ${ }^{* 1}$

Av. Dr. Arnaldo, 455 - Cerqueira César, Sao Paulo - SP, 01246-903 - sala 2364

hellenmartins@usp.br

Couto, Marcia Thereza ${ }^{2}$

Av. Dr. Arnaldo, 455 - Cerqueira César, Sao Paulo - SP, 01246-903 - sala 2165

marthet@usp.br

Daley, Kate ${ }^{3}$

Av. Dr. Arnaldo, 455 - Cerqueira César, Sao Paulo - SP, 01246-903 - sala 2364

drkatedaley@gmail.com

Rocha, Thais Izabel Ugeda ${ }^{4}$

Av. Dr. Arnaldo, 455 - Cerqueira César, Sao Paulo - SP, 01246-903 - sala 2364

thais.mentor@gmail.com

Aschar, Suzana Crismanis de Almeida Lopes ${ }^{5}$

Av. Dr. Arnaldo, 455 - Cerqueira César, Sao Paulo - SP, 01246-903 - sala 2364

suzana cal@yahoo.com.br

Santos, Daniela Vera Cruz dos ${ }^{6}$

Av. Dr. Arnaldo, 455 - Cerqueira César, Sao Paulo - SP, 01246-903 - sala 2364

dani.vera.cruz.santos@gmail.com

Claro, Heloísa Garcia ${ }^{7}$

Av. Dr. Arnaldo, 455 - Cerqueira César, Sao Paulo - SP, 01246-903 - sala 2364

heloisa.claro@usp.br 
Araya, Ricardo ${ }^{8}$

18 De Crespigny Park, London, SE5 8AF

ricardo.araya@kcl.ac.uk

Menezes, Paulo Rossi ${ }^{9}$

Av. Dr. Arnaldo, 455 - Cerqueira César, Sao Paulo - SP, 01246-903 - sala 2364

pmenezes@usp.br

*Corresponding author : hellenmartins@usp.br

\section{Abstract}

Background: Task-shifting and eHealth have been proposed as ways of approaching the mental health treatment gap in low and middle-income countries (LMIC). The Latin America Treatment \& Innovation Network in Mental Health (LATIN-MH) developed CONEMO (Emotional Control), a behavioral intervention used to treat depressive symptoms in a sample of patients with diabetes and hypertension in Sao Paulo, Brazil. The intervention uses task shifting and is delivered via a smartphone app. The effectiveness of this intervention was tested in two randomized trials in these countries.

Aim: This paper aims to present the protocol for a study that will explore perceived barriers and facilitators to implementing this intervention to help future scale-up.

Methods: We will conduct qualitative research with users of the CONEMO intervention and health professionals who participated directly and indirectly in the trial in Brazil. We will use semi-structured interviews, and we will adopt the Consolidated Framework for Implementation Research (CFIR) for data analysis.

Discussion: Task-shifting and eHealth are potentially important tools to help decrease the mental health treatment gap in Latin America. This study will increase our understanding of the factors which may facilitate or hinder the implementation of mobile behavioral mental health interventions, using taskshifting, within LMICs. Findings could be used in future design and planning to facilitate successful implementation and treatment. 
Keywords: Mental Health, Depression, Chronic diseases, Mobile Intervention, ehealth

$\underline{\text { Registration in Clinical Trials (www.clinical.trials.gov) }}$

NCT028406662

\section{Contributions to the literature}

- The research is important for the use of the Consolidated Framework for Implementation Research in the primary care context of low and middle-income countries, like the Brazil.

- Thesis findings will can contribute to think an effective implementation that decrease mental health treatment gap in primary care. 


\section{Authors' contributions}

$\mathrm{HC}$ and PM conceived the design of the study and drafted the protocol paper. MC contributed to the design of the study. All authors wrote, read, commented, and approved the final manuscript.

\section{Administrative information}

\begin{tabular}{|c|c|}
\hline Title $\{1\}$ & $\begin{array}{l}\text { Barriers and facilitators to implementing a mobile intervention } \\
\text { with task shifting in the treatment of depressive symptoms in } \\
\text { people with diabetes and/or hypertension in Brazil: A study } \\
\text { protocol using the CFIR. }\end{array}$ \\
\hline Trial registration $\{2 \mathrm{a}$ and $2 \mathrm{~b}\}$. & NCT028406662 \\
\hline Protocol version $\{3\}$ & 04-08-2020 - version 1.0 \\
\hline Funding $\{4\}$ & $\begin{array}{l}\text { Latin-MH received from the National Institute of Mental Health } \\
\text { NIMH a grant to develop and test the CONEMO intervention in } \\
\text { Brazil (1U19MH098780-01) } \\
\text { The qualitative study received an administrative supplement } \\
\text { (Grant Number: } 3 \mathrm{U} 19 \mathrm{MH} 098780--04 \mathrm{~S} 1 \text { ) from the National } \\
\text { Institute of Mental Health (NIMH) to conduct this qualitative } \\
\text { study that will evaluate facilitators and barriers of the Task } \\
\text { Shifting in the implementation of the CONEMO intervention. }\end{array}$ \\
\hline Author details $\{5 a\}$ & $\begin{array}{l}\text { 1. Doctorate student of the Department of Preventive Medicine, } \\
\text { Universidade de Sao Paulo - São Paulo - Brazil. Coordinator of } \\
\text { Shared Research at the Latin America Treatment \& Innovation } \\
\text { Network in Mental Health - Site 1. Av. Dr. Arnaldo, } 455 \text { - } \\
\text { Cerqueira César, Sao Paulo - SP, 01246-903. } \\
\text { 2. Associate Professor. Department of Preventive Medicine, } \\
\text { Universidade de Sao Paulo - São Paulo - Brazil. Av. Dr. } \\
\text { Arnaldo, } 455 \text { - 2nd floor - room } 2165 \\
\text { 3. Research at the Latin America Treatment \& Innovation } \\
\text { Network in Mental Health - Department of Preventive Medicine, } \\
\text { Department of Preventive Medicine, Universidade de Sao } \\
\text { Paulo - São Paulo - Brazil. Av. Dr. Arnaldo, } 455 \text { - Cerqueira } \\
\text { César, Sao Paulo - SP, 01246-903. } \\
\text { 4. Master student of the Department of Preventive Medicine, } \\
\text { Universidade de Sao Paulo - São Paulo - Brazil. Research at } \\
\text { the Latin America Treatment \& Innovation Network in Mental }\end{array}$ \\
\hline
\end{tabular}




\begin{tabular}{|c|c|}
\hline & $\begin{array}{l}\text { Health - Site 1. Av. Dr. Arnaldo, } 455 \text { - Cerqueira César, Sao } \\
\text { Paulo - SP, 01246-903. } \\
\text { 5. Master student of the Department of Preventive Medicine, } \\
\text { Universidade de Sao Paulo - São Paulo - Brazil. Research at } \\
\text { the Latin America Treatment \& Innovation Network in Mental } \\
\text { Health - Site 1. Av. Dr. Arnaldo, } 455 \text { - Cerqueira César, Sao } \\
\text { Paulo - SP, 01246-903. } \\
\text { 6. Master student of the Department of Preventive Medicine, } \\
\text { Universidade de Sao Paulo - São Paulo - Brazil. Coordinator of } \\
\text { Cost-Effectiveness at the Latin America Treatment \& Innovation } \\
\text { Network in Mental Health - Site 1. Av. Dr. Arnaldo, } 455 \text { - } \\
\text { Cerqueira César, Sao Paulo - SP, 01246-903 } \\
\text { 7. Professor at Universidade Estadual de Campinas - } \\
\text { UNICAMP, School of Nursing - R. Tessália Vieira de Camargo, } \\
\text { 126 - Cidade Universitária, Campinas - SP, 13083-887 } \\
\text { 8. Professor of Global Mental Health and Director of the } \\
\text { Centre for Global Mental Health and Primary Care Research, } \\
\text { Health Service and Population Research, Institute of } \\
\text { Psychiatry, Psychology, and Neuroscience, King's College } \\
\text { London, London, United Kingdom - } 18 \text { De Crespigny Park, } \\
\text { London, SE5 8AF9. } \\
\text { 9. Full Professor and Head of the Department of Preventive } \\
\text { Medicine, Universidade de Sao Paulo - São Paulo - Brazil. Av. } \\
\text { Dr. Arnaldo, 455 - Cerqueira César, Sao Paulo - SP, 01246-903 } \\
\text { - room 2364 }\end{array}$ \\
\hline $\begin{array}{l}\text { Name and contact information for } \\
\text { the trial sponsor }\{5 b\}\end{array}$ & $\begin{array}{l}\text { NIMH - National Institute of Mental Health } \\
\text { Source: } \\
\text { https://www.nimh.nih.gov/about/organization/gmh/globalhubs/i } \\
\text { ndex.shtml. }\end{array}$ \\
\hline Role of sponsor $\{5 c\}$ & $\begin{array}{l}\text { "NIMH established the Collaborative Hubs for International } \\
\text { Research on Mental Health (CHIRMH) to increase the research } \\
\text { base for mental health interventions in LMICs through the } \\
\text { integration of findings from translational, clinical, } \\
\text { epidemiological, and/or policy research. The program aims to } \\
\text { expand research activities in LMICs to provide the necessary } \\
\text { knowledge, tools, and sustainable research-based strategies } \\
\text { for use by government agencies, non-governmental } \\
\text { organizations, and health care institutions to reduce the mental } \\
\text { health treatment gap. Lessons learned from these contexts can } \\
\text { inform mental health service delivery in other low-resource } \\
\text { settings. As a group, awardees constitute a collaborative }\end{array}$ \\
\hline
\end{tabular}




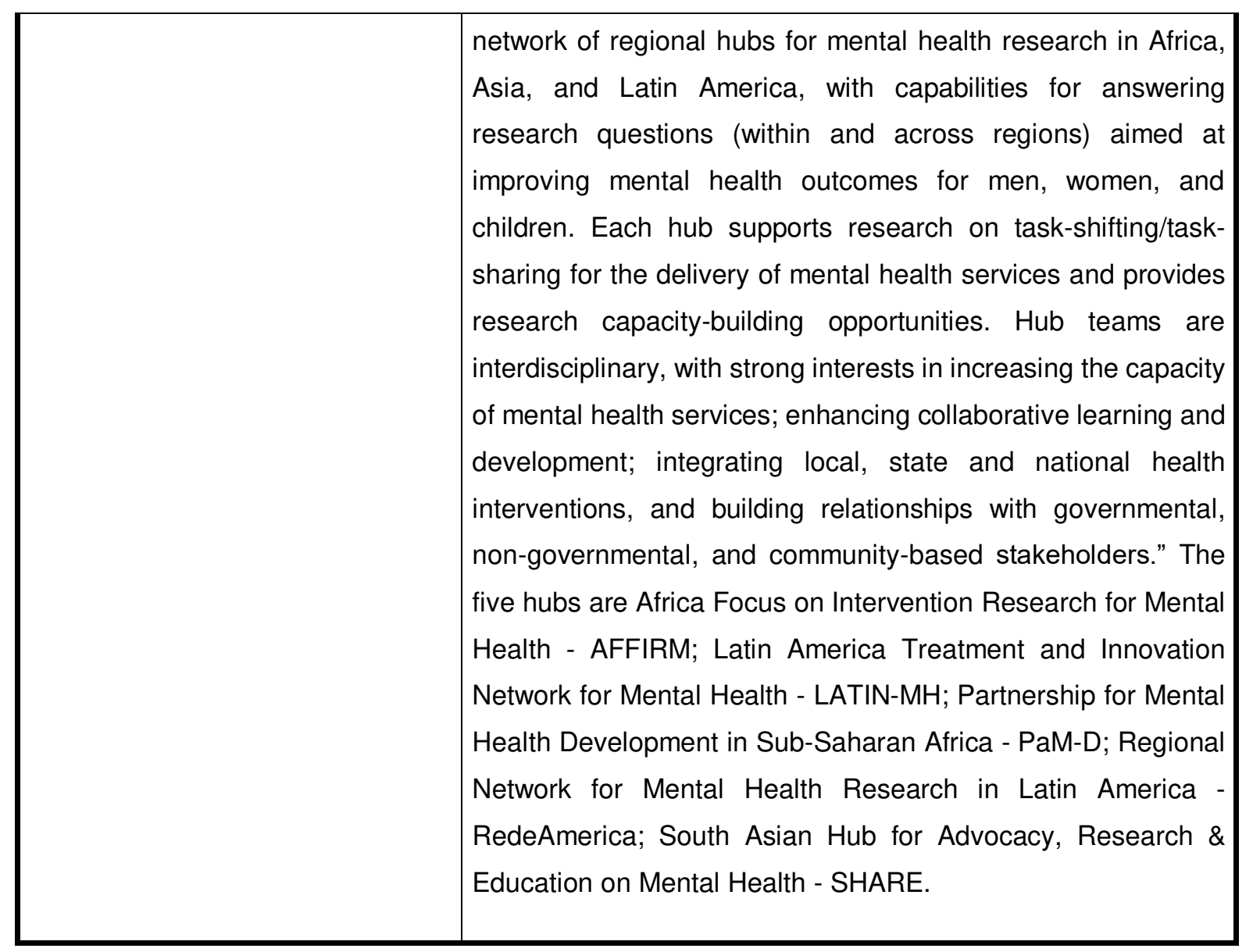

\section{Background}

Mental health problems often coexist with physical health difficulties. Rates of depression in populations with chronic disease are found to be higher than those in the general community. In Brazil, the prevalence rate of depression is $4.1 \%$, rising to $7.5 \%$ in those with hypertension and $8.6 \%$ in those with diabetes (Munhoz et al. 2016). The estimated prevalence of the major depressive disorder among adults in Sao Paulo is $9.4 \%$. In Brazil, almost $80 \%$ of those experiencing depression do not receive any treatment, and of those who do, the most common treatment is medication without follow-up (Lopes et al. 2016), which creates a mental health treatment gap.

Currently, there is an increasing global demand for mental health care and a lack of specialized human resources to meet this need in LMICs, especially within primary and secondary care. Evaluation of task-shifting in mental health settings has shown task exchange or delegation to be an effective strategy to 
cope with this demand (Agyapong et al. 2015). Active service user participation and the use of trained non-specialists could be essential tools to increase access to mental health care (Patel et al. 2010). Collaborative care for depression has also been found to be more effective than usual care, reinforcing the idea that integration among health professionals is likely better than clinical or educational guidelines alone (Patel et al. 2010).

Task-shifting is a strategy to delegate tasks usually performed by professionals with higher qualifications to people with a lower level of formal education or training (Verdeli 2016; Joshi et al. 2014). It creates a new workforce with specific training for particular tasks and offers a pragmatic response to health worker shortages (Joshi et al. 2014; Agyapong et al. 2015).

The Latin America Treatment and Innovation Network for Mental Health (LATIN-MH) has developed a mobile behavioral intervention (CONEMO) to treat depressive symptoms in patients with diabetes or hypertension, which utilizes task shifting. CONEMO is a low-intensity intervention that is based on behavioral activation and offered through a smartphone application. It comprises 18 sessions delivered three times a week for six weeks, with the support and monitoring of a Nurse Assistant. The Nurse Assistants (NAs) were recruited to be part of the task-shifting workforce and were responsible for teaching participants to use the CONEMO app, monitoring adherence to the intervention, helping participants with technological issues, and making telephone contacts to support and motivate participants. The NAs were trained and supervised by Psychologists. CONEMO is one of the first smartphone applications that offer psychoeducational intervention in mental health, developed and tested in LMICs. The clinical effectiveness of the response is being evaluated in two RCTs (Randomized Control Trials) comparing CONEMO to enhanced usual care.

If the CONEMO intervention is shown to be effective, it could be an essential tool in reducing the mental health treatment gap. To make this successful, it is important to assess the barriers and facilitators to implementation. This would enable necessary adjustments to be made to make the proposal viable and should help guarantee the best use of funds and resources.

To improve intervention success, it is important to adapt implementation strategies to the local context. Psychological interventions must be accepted by the patients and professionals involved in the process (Atif et al. 2016). 
Professional and user opinions, perceptions or understanding of intervention can influence implementation and outcome (Agyapong et al. 2016; Knowles et al. 2013). Efficacy of treatment is proven according to cultural sensitivity, contextual relevance, and public comprehension of the importance and use of this treatment (Patel et al. 2010).

\section{Aim}

This study aims to explore the perceived barriers and facilitators to implementing a smartphone delivered intervention with task shifting in the treatment of depressive symptoms in people with diabetes and or hypertension.

This protocol is part of the RCT and is linked to the "Shared Research Project" of the five HUBs funded by the National Institute of Mental Health. The mission of this shared research is to explore the implementation of task-shifting programs in mental health care settings in low and middle-income countries. The five research groups are working on qualitative research to investigate critical facilitators and barriers to their interventions, according to the singularities and context of those involved (Minayo \& de Souza Minayo, 2012). This paper focus on the results of the Sao Paulo branch of the shared research.

\section{Method}

We will conduct a cross-sectional qualitative study using semi-structured interviews to explore the barriers and facilitators to successful implementation of CONEMO, from a professional and user perspective.

\section{Sample}

We will interview individuals who either participated in the RCTs as a participant (patient of the health care system) or as health care professional involved directly or indirectly in the trial (Nurse Assistant or Manager). The interviewees range from patients from intervention groups, professionals, managers and policymakers invited to acquire their opinion about this proposal and others alike.

A total of 880 participants in the Sao Paulo RCT were recruited from 20 family health units (FHU), using cluster randomization. 
For this study, the user's sample constitution criteria will be nonprobabilistic, using the saturation theory to determine the final sample (Mason, 2010). This will enable us to reach a representative sample, with a diverse range of experience with the RCT process.

\section{Interviews Guide}

The semi-structured interviews will be based on a standard proposal from the "Collaborative HUBs Shared Research" to facilitate the comparison of data across HUBs. The proposal has been translated and culturally adapted by the Latin-MH research team.

There will be four interview templates, one for each participant category: participants from intervention, NAs, Managers, and policymakers. A summary of the RCT proposal will be presented to policymakers before the interview since they did not participate in the RCT.

\section{Procedures}

\section{Participant selection}

The following criteria will be used for selecting participants, respecting the proportion in each category:

a) Adherence to intervention:

i) No adherence: participants who completed only the training session;

ii) Medium adherence: participants who completed up to $50 \%$ of sessions;

iii) High adherence: participants who completed more than $50 \%$ of sessions.

b) Geographic area: participants from all FHU's in Sao Paulo

The list of relevant characteristics and the number of participants required will be sent to the Data Center Coordination (DCC). The DCC will then provide a list of potential participants. We will use the software Stata 13 to carry out a selection by drawing, respecting the established criteria. The health professionals will be selected based on the experience of the research team based on their engagement. 


\section{Data Collection}

Participants will be contacted by phone, and those interested in taking part will be interviewed by the research team, who will give a detailed explanation about the research, allowing questions, and obtaining informed consent. Interviews will be face-to-face, recorded using a portable voice recorder, and subsequently transcribed verbatim. Interviews will take place confidentially, in a private room, free from noise and distraction.

\section{Data Analysis}

After transcription, interviews will be analyzed using QSR International Pty Ltd's N-VIVO 12 Pro For Windows, software to support the organization and analysis of qualitative interview data.

The Consolidated Implementation Research Framework (CFIR) will be used to guide the analysis to understand the facilitators and barriers to implementation. The CFIR is a conceptual explanatory framework used to identify constructs and mechanisms which may influence implementation and effectiveness (Damschroder et al. 2009).

\section{Discussion}

Task-shifting and e-health are viable strategies for increasing access to mental health care and reducing the treatment gap. CONEMO is one such intervention, and if shown to be effective, it would be fundamental to investigate which aspects can facilitate or hinder its implementation.

Lawton et al. (2015) highlight that the emotional aspects of people who work in the trial/intervention should be taken into account to ensure they are supported and able to conduct their tasks adequately (Lawton et al. 2015). Emotional challenges associated with conflicts between research and clinical activities may be stressful. Professional's opinions and perceptions must be taken into account regarding any difficulties faced, for example, in handling the intervention, workload, or relationship with participants. 
This study is fundamental to help identify and understand the facilitators and barriers to implementation of the CONEMO intervention from the perspective of those involved. Analyzing the social, cultural and territorial representations related to this intervention will allow the design of a customized proposal, with higher chances of being accepted and, therefore, successful.

\section{Trial Status}

Recruitment closed.

\section{List of abbreviations}

$\begin{array}{ll}\text { CONEMO } & \text { Emotional Control } \\ \text { CONEP } & \text { National Commission for Ethics in Research } \\ \text { DSMB } & \text { Global Mental Health Data and Safety Monitoring Board } \\ \text { FHU } & \text { Family Health Unit } \\ \text { HUBs } & \text { Collaborative Hubs Shared Research } \\ \text { LATIN-MH } & \text { Latin America Treatment \& Innovation Network in Mental } \\ & \text { Health } \\ \text { NIMH } & \text { National Institute of Mental Health - USA } \\ \text { Na } & \text { Nurse Assistant } \\ \text { RCT } & \text { Randomized Control Trial } \\ \text { TCLE } & \text { Free Term Explained } \\ \text { U19 } & \text { Collaborative Hubs for International Research on Mental } \\ & \text { Health (U19). }\end{array}$

\section{References}

Agyapong, V.I.O. et al., 2015. Task shifting--Ghana's community mental health workers' experiences and perceptions of their roles and scope of practice. Global health action, 8, p.28955.

Agyapong, V.I.O., Farren, C. \& McAuliffe, E., 2016. Improving Ghana's mental healthcare through task-shifting- psychiatrists and health policy directors perceptions about government's commitment and the role of community mental health workers. Globalization and health, 12(1). Available at: http://dx.doi.org/10.1186/s12992-016-0199-z.

Damschroder, L.J. et al., 2009. Fostering implementation of health services research findings into practice: a consolidated framework for advancing implementation science. Implementation science: IS, 4, p.50.

Joshi, R. et al., 2014. Task shifting for non-communicable disease management in low and middle income countries--a systematic review. PloS one, 9(8), p.e103754. 
Knowles, S.E. et al., 2013. Better together? a naturalistic qualitative study of inter-professional working in collaborative care for co-morbid depression and physical health problems. Implementation science: IS, 8, p.110.

Lopes, C.S. et al., 2016. Inequities in access to depression treatment: results of the Brazilian National Health Survey - PNS. International journal for equity in health, 15(1). Available at: http://dx.doi.org/10.1186/s12939-016-0446-1.

Mason, Mark. (2010). Sample Size and Saturation in PhD Studies Using Qualitative Interviews. Forum Qualitative Sozialforschung / Forum: Qualitative Social Research. 11.

Minayo, M.C. de S. \& de Souza Minayo, M.C., 2012. Análise qualitativa: teoria, passos e fidedignidade. Ciencia \& saude coletiva, 17(3), pp.621-626.

Munhoz, T.N. et al., 2016. A nationwide population-based study of depression in Brazil. Journal of affective disorders, 192, pp.226-233.

Patel, V. et al., 2010. Reducing the treatment gap for mental disorders: a WPA survey. World psychiatry: official journal of the World Psychiatric Association, 9(3), pp.169-176.

Verdeli, H., 2016. Global Mental Health: An Introduction. Journal of clinical psychology, 72(8), pp.761-765.

\section{Declarations}

\section{Ethics approval and consent to participate}

The subproject was approved by the following institutions:

\section{Brazil:}

CEP - Coordination of Ethics in Research da Faculdade de Medicina da Universidade de Sao Paulo, number 2,348,319, on August 30, 2017

CONEP - National Commission for Ethics in Research (Comissão Nacional de Ética em Pesquisa, federal government), 2,400,542 on October 30, 2017;

SMS CEP ( number: 2,636,669 on May 4, 2018)

\section{United States:}

DSMB - Global Mental Health Data and Safety Monitoring Board (DSMB) on January 8, 2018, for the Sao Paulo qualitative study. 


\section{Consent for publication}

Not applicable.

\section{Availability of data and materials}

The dataset (which includes individual transcripts and audios) is not publicly available due to confidentiality policies.

\section{Competing interests}

The authors declare that they have no competing interests.

\section{Funding}

Latin-MH received from the National Institute of Mental Health NIMH a grant to develop and test the CONEMO intervention (1U19MH098780-01)

The qualitative study received an administrative supplement (Grant Number: 3U 19MH098780--04S1) from the National Institute of Mental Health (NIMH) to conduct this qualitative study that will evaluate facilitators and barriers of the Task Shifting in the implementation of the CONEMO intervention.

\section{Acknowledgements}

We would like to thank the Research Assistants, Ariane de Souza Nascimento and Messias Mendes da Silva for their commitment in collecting the subproject data and facing challenges inherent in field work with ethics and responsibility. Thanks to George Abud Scotton from the Data Center for helping with participants selection.

\section{Authors' contributions}

HC, PM and RA conceived the design of the study and drafted the protocol paper.

$M C$ contributed to the design of the study.

$\mathrm{KD}, \mathrm{TR}, \mathrm{SA}$, DS and HGC wrote, read, commented and approved the final manuscript.

\section{Authors' information (optional)}

1. Doctorate student of the Department of Preventive Medicine of the Universidade de São Paulo - Brazil. Coordinator of Shared Research at the Latin America Treatment \& Innovation Network in Mental Health - Site 
1. Av. Dr. Arnaldo, 455 - Cerqueira César, Sao Paulo - SP, 01246-903 room 2364

2. Associate Professor. Department of Preventive Medicine, of the Universidade de São Paulo - Brazil. Av. Dr. Arnaldo, 455 - 2nd floor - room 2165

3. Research at the Latin America Treatment \& Innovation Network in Mental Health - Site 1. Av. Dr. Arnaldo, 455 - Cerqueira César, Sao Paulo - SP, 01246-903 - room 2364

4. Master student of the Department of Preventive Medicine of the of the Universidade de São Paulo - Brazil. Research at the Latin America Treatment \& Innovation Network in Mental Health - Site 1. Av. Dr. Arnaldo, 455 - Cerqueira César, Sao Paulo - SP, 01246-903 - room 2364

5. Master student of the Department of Preventive Medicine of the of the Universidade de São Paulo - Brazil. Research at the Latin America Treatment \& Innovation Network in Mental Health - Site 1. Av. Dr. Arnaldo, 455 - Cerqueira César, Sao Paulo - SP, 01246-903 - room 2364

6. Master student of the Department of Preventive Medicine of the of the Universidade de São Paulo - Brazil. Coordinator of Cost-Effectiveness at the Latin America Treatment \& Innovation Network in Mental Health - Site 1. Av. Dr. Arnaldo, 455 - Cerqueira César, Sao Paulo - SP, 01246-903 room 2364

7. Professor at Universidade Estadual de Campinas - UNICAMP, School of Nursing. Campinas - Brazil. Coordinator of Data Center at the Latin America Treatment \& Innovation Network in Mental Health - Site 1. Av. Dr. Arnaldo, 455 - Cerqueira César, Sao Paulo - SP, 01246-903 - room 2364

8. Professor of Global Mental Health and Director of the Centre for Global Mental Health and Primary Care Research, Health Service and Population Research, Institute of Psychiatry, Psychology, and Neuroscience, King's College London, London, United Kingdom - 18 De Crespigny Park, London, SE5 8AF9. 
9. Full Professor and Head of the Department of Preventive Medicine, of the Universidade de São Paulo - Brazil. Av. Dr. Arnaldo, 455 - Cerqueira César, Sao Paulo - SP, 01246-903 - room 2364

\section{Endnotes}

a- "NIMH established the Collaborative Hubs for International Research on Mental Health (CHIRMH) to increase the research base for mental health interventions in LMICs through the integration of findings from translational, clinical, epidemiological and/or policy research. The program aims to expand research activities in LMICs to provide the necessary knowledge, tools, and sustainable research-based strategies for use by government agencies, non-governmental organizations, and health care institutions to reduce the mental health treatment gap. Lessons learned from these contexts can inform mental health service delivery in other lowresource settings. As a group, awardees constitute a collaborative network of regional hubs for mental health research in Africa, Asia, and Latin America, with capabilities for answering research questions (within and across regions) aimed at improving mental health outcomes for men, women, and children. Each hub supports research on task-shifting/tasksharing for the delivery of mental health services and provides research capacity-building opportunities. Hub teams are interdisciplinary, with strong interests in increasing the capacity of mental health services; enhancing collaborative learning and development; integrating local, state and national health interventions, and building relationships with governmental, non-governmental, and community-based stakeholders." The five hubs are Africa Focus on Intervention Research for Mental Health - AFFIRM; Latin America Treatment and Innovation Network for Mental Health - LATIN-MH; Partnership for Mental Health Development in SubSaharan Africa - PaM-D; Regional Network for Mental Health Research in Latin America - RedeAmerica; South Asian Hub for Advocacy, Research \& Education on Mental Health - SHARE. Source: https://www.nimh.nih.gov/about/organization/gmh/globalhubs/index.shtml 


\section{Supplementary Files}

This is a list of supplementary files associated with this preprint. Click to download.

- SupplementaryFileChecklist.pdf 